\section{UJMM

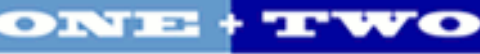

\section{Undergraduate Journal of Mathematical}

Modeling: One + Two

Volume 3 | 2010 Fall

Article 21

2010

\title{
Motion of a Pendulum
}

Jared Wynn

University of South Florida

Advisors:

Arcadii Grinshpan, Mathematics and Statistics

Dennis Killinger, Physics

Problem Suggested By: Dennis Killinger

Follow this and additional works at: https://digitalcommons.usf.edu/ujmm

Part of the Mathematics Commons

UJMM is an open access journal, free to authors and readers, and relies on your support:

Donate Now

\section{Recommended Citation}

Wynn, Jared (2010) "Motion of a Pendulum," Undergraduate Journal of Mathematical Modeling: One + Two: Vol. 3: Iss. 1, Article 9.

DOI: http://dx.doi.org/10.5038/2326-3652.3.1.21

Available at: https://digitalcommons.usf.edu/ujmm/vol3/iss1/21 


\section{Motion of a Pendulum}

\section{Abstract}

The objective of this project is to derive and solve the equation of motion for a pendulum swinging at small angles in one dimension. The pendulum may be either a simple pendulum like a ball hanging from a string or a physical pendulum like a pendulum on a clock. For simplicity, we only considered small rotational angles so that the equation of motion becomes a harmonic oscillator.

\section{Keywords}

Pendulum, Harmonic Oscillator, Periodic Motion

\section{Creative Commons License}

\section{(c) (1) $9 \odot$}

This work is licensed under a Creative Commons Attribution-Noncommercial-Share Alike 4.0 License.

\section{Erratum}

This article was previously called Article 21. 


\section{TABLE OF CONTENTS}

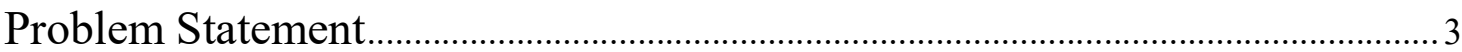

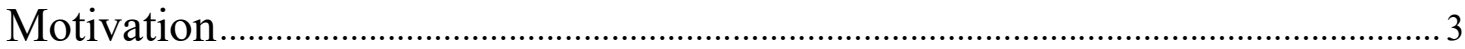

Mathematical Description and Solution Approach ….............................................. 3

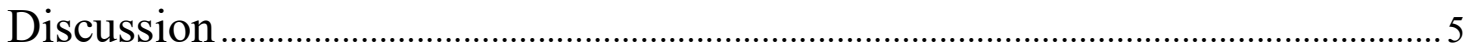

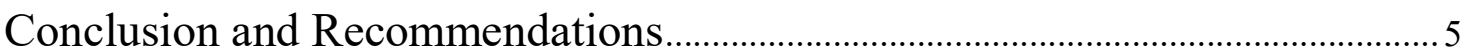

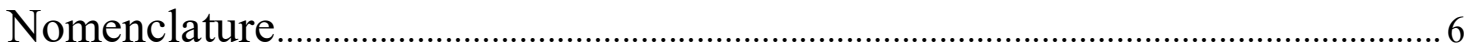




\section{PROBLEM STATEMENT}

The goal of this project is to accurately describe the motion of pendulums in terms of calculus and physics. There are two types of pendulums. A simple pendulum is a mass dangling at the bottom of a string. A physical pendulum is a rigid hanging object which oscillates about a stationary point that is not located at its center of mass. We shall derive and solve the differential equation of motion of pendulums. In order to simplify the differential equation for the motion of a pendulum, we shall assume that the pendulum is swinging at small angles and only swinging back and forth in one dimension rather than swinging roundly. Throughout this project, we shall ignore the effects of air resistance and friction.

\section{MOTIVATION}

People use pendulums in some mechanical systems such as grandfather clocks, car alarm systems, and seismographic instruments to measure the magnitude of physical shock. Many simple devices are also pendulums. Such everyday devices include swings on a swing set and rope swings suspended from a tree branch. The best example of a physical pendulum is the grandfather clock arm because it is rigid and pivots from a stationary point. It is therefore worthwhile to characterize a pendulum's motion as it swings back and forth.

\section{MATHEMATICAL DESCRIPTION AND SOLUTION APPROACH}

Newton's second law of motion, namely $F=m a$, can be used to mathematically describe the motion of a pendulum. In terms of rotation, Newton's second law becomes the equation

$$
\tau=I \alpha
$$


where $I$ is the moment of inertia of the pendulum, $\alpha$ is the angular acceleration, and $\tau$ is the torque acting on the pendulum. Using Newton's second law in angular form, we shall derive the equation of motion for a pendulum.

Since torque is directly proportional to the angle of rotation $\theta$, we get

$$
\tau=-c \sin \theta
$$

where $c$ is the torque of the pendulum at a $90^{\circ}$ angle. If the pendulum is a simple pendulum or a physical pendulum, we get

$$
c=m g d
$$

where $m g$ is the normal force, and $d$ is the center of mass. Since the angular acceleration $\alpha$ is the change in angular velocity $\frac{d \theta(t)}{d t}$, we get

$$
\alpha(t)=\frac{d^{2} \theta(t)}{d t^{2}}
$$

so

$$
-c \sin \theta(t)=I \alpha(t)=I \frac{d^{2} \theta(t)}{d t^{2}}
$$

When the angle $\theta$ is small, the function $\sin \theta$ is approximately $\theta$. Therefore, for small angles, the pendulum satisfies the equation

$$
\left(-\frac{c}{I}\right) \theta(t)=\frac{d^{2} \theta(t)}{d t^{2}}
$$

Assuming the initial velocity is zero, $\theta^{\prime}(0)=0$, the general solution to (6) is

$$
\theta(t)=A \cos \left(\sqrt{\frac{c}{I}} t\right)=A \cos \left(\sqrt{\frac{m g d}{I}} t\right)
$$

where $A$ is the amplitude or maximum angular position of the object. Since the sine function is $2 \pi$ periodic, the amount of time needed for the pendulum to swing one full period (back and forth) is 


$$
T=2 \pi \sqrt{I / c}=2 \pi \sqrt{I / m g d}
$$

and the frequency of the pendulum is

$$
f=\frac{1}{T}=\frac{1}{2 \pi} \sqrt{m g d / I} \text {. }
$$

\section{DISCUSSION}

Note that equation (1) is essentially

$$
-\omega^{2} \theta=\frac{d^{2} \theta}{d t^{2}}
$$

the equation for a harmonic oscillator. Also take note that as $\theta$ grows larger,equation (6) becomes less accurate since $\theta$ does not approximate $\sin \theta$ very well.Instead, one has to deal with the equation $-\frac{c}{I} \sin (\theta)=\frac{d^{2} \theta}{d t^{2}}$ which cannot be solved in terms of elementary functions. Therefore one must use special functions or numerical methods to solve the equation of motion of a pendulum swinging at larger angles.

\section{CONCLUSION AND RECOMMENDATIONS}

We recommend further studies on the equation of motion for a pendulum. For example, one could do a study on the equation of motion of a pendulum for pendulums that swing at larger angles. Furthermore, one could also incorporate air resistance and friction in the equation of motion of the pendulum. One could even study the motion of a pendulum moving in two dimensions instead of a pendulum just moving back and forth. 


\section{NOMENCLATURE}

\begin{tabular}{|clc|}
\hline Symbol & Description & Units \\
\hline$F$ & Force & $\mathrm{N}$ \\
\hline$m$ & Mass & $\mathrm{kg}$ \\
\hline$a$ & Acceleration & $\frac{\mathrm{m}}{\mathrm{s}^{2}}$ \\
\hline$c$ & Torque at $90^{\circ}$ & $\mathrm{Nm}$ \\
\hline$\alpha$ & Angular Acceleration & $\frac{\mathrm{rad}}{\mathrm{s}^{2}}$ \\
\hline$I$ & Moment of Inertia & $\frac{\mathrm{kg}}{\mathrm{m}^{2}}$ \\
\hline$\theta$ & Angle & $\mathrm{deg}$ \\
\hline$t$ & Time & $\mathrm{s}$ \\
\hline$D$ & Distance & $\mathrm{m}$ \\
\hline$A$ & Amplitude & $\mathrm{deg}$ \\
\hline$T$ & Period & $\mathrm{s}$ \\
\hline$f$ & Frequency & $\frac{\mathrm{rad}}{\mathrm{s}}$ \\
\hline
\end{tabular}

\section{REFERENCES}

Baker, Gregory and James Blackburn. The Pendulum: A Physics Case Study. Oxford University Press, 2005.

Larson, Ron, Robert Hostetler and Bruce Edwards. Calculus. 8th Edition. Boston, MA: Houghton Mifflin Company, 2005. 